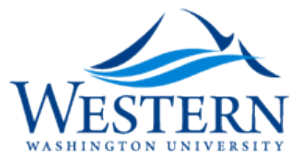

Western Washington University Western CEDAR

$1-2003$

\title{
On the Location of Critical Points of Polynomials
}

Branko Ćurgus

Western Washington University, branko.curgus@wwu.edu

Vania Mascioni

Follow this and additional works at: https://cedar.wwu.edu/math_facpubs

Part of the Mathematics Commons

\section{Recommended Citation}

Curgus, Branko and Mascioni, Vania, "On the Location of Critical Points of Polynomials" (2003). Mathematics. 7. https://cedar.wwu.edu/math_facpubs/7

This Article is brought to you for free and open access by the College of Science and Engineering at Western CEDAR. It has been accepted for inclusion in Mathematics by an authorized administrator of Western CEDAR. For more information, please contact westerncedar@wwu.edu. 


\title{
ON THE LOCATION OF CRITICAL POINTS OF POLYNOMIALS
}

\author{
BRANKO ĆURGUS AND VANIA MASCIONI
}

(Communicated by N. Tomczak-Jaegermann)

\begin{abstract}
Given a polynomial $p$ of degree $n \geq 2$ and with at least two distinct roots let $Z(p)=\{z: p(z)=0\}$. For a fixed root $\alpha \in Z(p)$ we define the quantities $\omega(p, \alpha):=\min \{|\alpha-v|: v \in Z(p) \backslash\{\alpha\}\}$ and $\tau(p, \alpha):=$ $\min \left\{|\alpha-v|: v \in Z\left(p^{\prime}\right) \backslash\{\alpha\}\right\}$. We also define $\omega(p)$ and $\tau(p)$ to be the corresponding minima of $\omega(p, \alpha)$ and $\tau(p, \alpha)$ as $\alpha$ runs over $Z(p)$. Our main results show that the ratios $\tau(p, \alpha) / \omega(p, \alpha)$ and $\tau(p) / \omega(p)$ are bounded above and below by constants that only depend on the degree of $p$. In particular, we prove that $(1 / n) \omega(p) \leq \tau(p) \leq(1 / 2 \sin (\pi / n)) \omega(p)$, for any polynomial of degree $n$.
\end{abstract}

\section{INTRODUCTION}

The attempt to locate the roots of polynomials has a long and golden history, from the Galois-Abel theory to present day numerical methods, and yet the inherent (nonlinear) difficulties have hampered the investigation of the geometrical side of the subject. The classical literature presents some well-rounded results on the relationship between the roots of a polynomial and those of its derivative, but no groundbreaking progress has been registered since then. After seminal work by Lucas, Grace and Haewood, J. L. Walsh has been at the forefront of this research for a good part of the last century, and his work (and not only his) is summarized in his excellent monograph [7]. After this, even more recent surveys (see for example the excellent chapter on polynomials in [2], or [1]) do not display any essential advance in knowledge (in terms of the geometry of the roots) compared to the standard reference book by Marden [3], which summarizes most of the classical work in the area. Generally speaking, it seems that the insight provided by Lucas' Theorem (which says that the convex hull of the roots of $p$ contains all the roots of $p^{\prime}$ ) and a handful of other classical results are still the best that the modern researcher can rely on.

In this paper we study how two specific quantities measured on a polynomial compare to each other. We present a local and a global version of these quantities (see Proposition 2 and Theorem 4 below). At the global level, one of the quantities (which we call $\omega(p)$, see (4) below), measures the smallest distance between any two distinct roots of a polynomial $p$. The quantity $\omega(p)$ is referred to as $\operatorname{sep}(p)$ in Mignotte's book [4], where an entire section is devoted to the separation of the roots

Received by the editors July 10, 2001 and, in revised form, September 4, 2001.

2000 Mathematics Subject Classification. Primary 30C15; Secondary 26C10.

Key words and phrases. Roots of polynomials, critical points of polynomials, separation of roots. 
of a polynomial. Mignotte includes the proof of some delicate estimates involving $\omega(p)$ but apparently no connection is made with the other quantity $\tau(p)$ (see (5) below) which we study in this paper. The quantity $\tau(p)$ measures the smallest distance between any root of $p$ and any of the "new" roots of the derivative $p^{\prime}$ (that is, the roots that are not already roots of $p$ ). While the first inequality in Proposition 2 and Theorem 4 is closely related to a result of Walsh [7], what we found interesting was the discovery that the quantities $\tau(p)$ and $\omega(p)$ are actually (loosely speaking) proportional. Moreover, the bounds in Theorem 4 are the best possible. To keep the exposition self-contained, and also because the explicit definitions of $\omega(p)$ and $\tau(p)$ are absent from Walsh's writings, we will provide a new and more direct proof of the first inequality as well. The second inequality in Theorem 4 contained a surprise, in that its proof seemed to flow smoothly until we realized that a special case of polynomials of degree $n=5$ escaped the direct power of known techniques, and this is why the work needed to cover the gap takes on the larger part of this proof. While we duly apologize for the brute force approach to this special case, we believe that the difficulty is a symptom of the number of truths that still remain to be uncovered in the subject.

\section{The INEQUALITIES}

By $\mathbb{C}$ we denote the set of all complex numbers. For $w \in \mathbb{C}$ and $r>0$ by $D(w, r)$ we denote the closed disk centered at $w$ with radius $r$; that is, $D(w, r)=\{z \in \mathbb{C}$ : $|z-w| \leq r\}$. Sometimes we shall use the expression "circle $D(w, r)$ " to refer to the boundary $\{z \in \mathbb{C}:|z-w|=r\}$ of $D(w, r)$. For two distinct complex numbers $u$ and $v$, by $\ell(u, v)$ we denote the line passing through $u$ and $v$. For a polynomial $p$, we define $Z(p)$ to be the set of all roots of $p$.

We are going to make repeated use of the following lemma which is a special case of the famous Two-Circle Theorem due to Walsh [7, Theorem 1, p. 59] (also see $[3$, Exercise $(19,4)]$ and [5] for an interesting alternate discussion). We quote the version we need for easy reference:

Lemma 1. Let $\alpha$ be any given point in the complex plane and $p$ a polynomial of degree $n$. Let $n_{1}$ roots of $p$ lie in the disk $D\left(\alpha, r_{1}\right)$, and let the other $n_{2}=n-n_{1}$ roots of $p$ lie outside or on the circle $D\left(\alpha, r_{2}\right)$. Then, if $r=\left(n_{1} r_{2}-n_{2} r_{1}\right) / n>r_{1}$, we have that exactly $n_{1}-1$ roots of $p^{\prime}$ lie in $D\left(\alpha, r_{1}\right)$ and exactly $n_{2}$ lie outside or on the circle $D(\alpha, r)$.

Let $p$ be a polynomial and assume that $p$ has at least two distinct roots, or equivalently, $Z\left(p^{\prime}\right) \backslash Z(p) \neq \emptyset$. Let $\alpha$ be a root of $p$. Define

$$
\omega(p, \alpha):=\min \{|\alpha-v|: v \in Z(p) \backslash\{\alpha\}\}
$$

and

$$
\tau(p, \alpha):=\min \left\{|\alpha-v|: v \in Z\left(p^{\prime}\right) \backslash\{\alpha\}\right\} .
$$

In the following proposition we prove two inequalities for these quantities. The first inequality in (3) below was obtained by Walsh (see [7, $\S 3.1 .1$, Corollary to Theorem 1]) as a corollary to his Two-Circle Theorem. Here we give a simpler direct proof.

Proposition 2. Let $p$ be a polynomial of degree $n, n>2$, and assume that $p$ has at least two distinct roots. Let $\alpha$ be a root of $p$ with multiplicity $k_{\alpha}, 1 \leq k_{\alpha} \leq n-2$. 
Then

$$
\frac{k_{\alpha}}{n} \omega(p, \alpha) \leq \tau(p, \alpha) \leq \frac{1}{\sin \left(\frac{\pi}{n-k_{\alpha}}\right)} \omega(p, \alpha) .
$$

If $n \geq 2$ and $k_{\alpha}=n-1$, then $\tau(p, \alpha)=\frac{n-1}{n} \omega(p, \alpha)$.

Proof. Fix $\alpha_{1} \in Z(p)$. To prove the first inequality we only need to consider the case when $\omega\left(p, \alpha_{1}\right)>\tau\left(p, \alpha_{1}\right)$. This assumption implies that

$$
\tau\left(p, \alpha_{1}\right)=\min \left\{\left|\alpha_{1}-v\right|: v \in Z\left(p^{\prime}\right) \backslash Z(p)\right\} .
$$

Let $k_{1}$ be the multiplicity of $\alpha_{1}$, and let $\alpha_{2}, \ldots, \alpha_{m}$ be all the other roots of $p$ (each with its corresponding multiplicity $\left.k_{j}, j=2, \ldots, m\right)$. Let $z_{0} \in Z\left(p^{\prime}\right) \backslash Z(p)$ be such that $\left|\alpha_{1}-z_{0}\right|=\tau\left(p, \alpha_{1}\right)$. Then we have

$$
0=\frac{p^{\prime}\left(z_{0}\right)}{p\left(z_{0}\right)}=\sum_{j=1}^{m} \frac{k_{j}}{z_{0}-\alpha_{j}}=\frac{k_{1}}{z_{0}-\alpha_{1}}+\sum_{j=2}^{m} \frac{k_{j}}{z_{0}-\alpha_{j}},
$$

and hence

$$
\frac{k_{1}}{\tau\left(p, \alpha_{1}\right)} \leq \sum_{j=2}^{m} \frac{k_{j}}{\left|z_{0}-\alpha_{j}\right|}
$$

Consider that for every $j \in\{2, \ldots, m\}$ we have

$$
\left|z_{0}-\alpha_{j}\right| \geq\left|\alpha_{1}-\alpha_{j}\right|-\left|z_{0}-\alpha_{1}\right| \geq \omega\left(p, \alpha_{1}\right)-\tau\left(p, \alpha_{1}\right),
$$

and therefore

$$
\frac{k_{1}}{\tau\left(p, \alpha_{1}\right)} \leq \frac{n-k_{1}}{\omega\left(p, \alpha_{1}\right)-\tau\left(p, \alpha_{1}\right)}
$$

which is equivalent to

$$
\frac{k_{1}}{n} \omega\left(p, \alpha_{1}\right) \leq \tau\left(p, \alpha_{1}\right) .
$$

Next we prove the second inequality in (3). Let $n>2$ and fix $\alpha=\alpha_{1} \in Z(p)$ and let $\alpha_{2} \in Z(p)$ be such that $\omega\left(p, \alpha_{1}\right)=\left|\alpha_{1}-\alpha_{2}\right|>0$. Let $q=n+1-k_{1}-k_{2}$, where $k_{1}$ and $k_{2}$ are the multiplicities of $\alpha_{1}$ and $\alpha_{2}$ and $k_{1} \leq n-2$. Let $K$ be the segment joining $\alpha_{1}$ and $\alpha_{2}$, and define the star-shaped region $S(K, \pi / q)$ to be the set of all points $w$ in $\mathbb{C}$ for which the angle at $w$ of the triangle with corners $w, \alpha_{1}, \alpha_{2}$ is greater or equal to $\pi / q$. By a result of Marden (see [3, Ex. (25,1)]), $p^{\prime}$ has at least one root $u \neq \alpha_{1}$ in the region $S(K, \pi / q)$. So, we immediately have that $\tau\left(p, \alpha_{1}\right) \leq\left|\alpha_{1}-u\right|$. Now, it is a simple exercise in polar coordinates to determine that a point $z$ of $S(K, \pi / q)$ that is most distant from $\alpha_{1}$ satisfies $\left|\alpha_{1}-z\right|=(\sin (\pi / q))^{-1}\left|\alpha_{1}-\alpha_{2}\right|$, and this readily implies

$$
\tau\left(p, \alpha_{1}\right) \leq\left|\alpha_{1}-u\right| \leq \frac{1}{\sin \left(\frac{\pi}{q}\right)} \omega\left(p, \alpha_{1}\right) \leq \frac{1}{\sin \left(\frac{\pi}{n-k_{1}}\right)} \omega\left(p, \alpha_{1}\right) .
$$

This proves (3). The last statement is easily verified since in this case $p(z)=$ $(z-\alpha)^{n-1}(z-\beta)$ and consequently $\omega(p, \alpha)=|\alpha-\beta|$ and $\tau(p, \alpha)=\frac{n-1}{n}|\alpha-\beta|$. The proposition is proved. 
Lucas' Theorem states that all the roots of $p^{\prime}$ lie in the convex hull of the roots of $p$, the so-called Lucas polygon of $p$. The importance of the first inequality in (3) is that it provides an improvement to Lucas' Theorem, which we state in the following corollary. This improvement was observed by Walsh in $[7, \S 3.4]$, but it does not appear in the books [3], [2] and [1]. The idea behind this improvement is extensively used in the proof of Theorem 4 below.

Corollary 3. Let $p$ be a polynomial of degree $n, n \geq 2$, and assume that $p$ has at least two distinct roots. Let $\alpha$ be a root of $p$ with multiplicity $k_{\alpha}$. The set $Z\left(p^{\prime}\right) \backslash Z(p)$ is contained in the "Swiss cheese"-like region obtained by removing the interiors of all the disks $D(\alpha, \tau(p, \alpha)), \alpha \in Z(p)$, from the Lucas polygon of $p$.

Next we define the global analogues of the quantities defined in (1) and (2). Let $p$ be a polynomial of degree $n, n \geq 2$, and assume that $p$ has at least two distinct roots. Define

$$
\omega(p):=\min \{|w-v|: w, v \in Z(p), w \neq v\}
$$

and

$$
\tau(p):=\min \left\{|w-v|: w \in Z(p), v \in Z\left(p^{\prime}\right) \backslash\{w\}\right\} .
$$

Clearly, $\tau(p)=\min \{\tau(p, \alpha): \alpha \in Z(p)\}$ and $\omega(p)=\min \{\omega(p, \alpha): \alpha \in Z(p)\}$.

Theorem 4. Let $p$ be a polynomial of degree $n, n \geq 2$, and assume that $p$ has at least two distinct roots. Then

$$
\frac{1}{n} \omega(p) \leq \tau(p) \leq \frac{1}{2 \sin (\pi / n)} \omega(p) .
$$

Remark 5. The inequality (6) is the best possible. This follows from the following two examples: for the polynomial $p(z)=z^{n-1}(z-1)$ we have $\omega(p)=1$ and $\tau(p)=\frac{1}{n}$, and for the polynomial $p(z)=z^{n}-1$ we have $\omega(p)=2 \sin (\pi / n)$ and $\tau(p)=1$.

Proof of Theorem 4. The first inequality follows immediately from Proposition 2. Indeed, letting $\alpha \in Z(p)$ be such that $\tau(p, \alpha)=\tau(p)$, (3) immediately implies

$$
\frac{1}{n} \omega(p) \leq \frac{1}{n} \omega(p, \alpha) \leq \tau(p, \alpha)=\tau(p) .
$$

Next we prove the second inequality in (6). Let $z_{1}, z_{2} \in Z(p)$ be such that $\omega(p)=\left|z_{1}-z_{2}\right|>0$. By the Grace-Heawood Theorem (see [3, Theorem (23,1)]), $p^{\prime}$ must have a root in the closed disk $D(c, r)$, where

$$
c=\frac{z_{1}+z_{2}}{2}, \quad r=\frac{\left|z_{1}-z_{2}\right|}{2} \cot \left(\frac{\pi}{n}\right) \text {. }
$$

Let $w \in D(c, r)$ be such that $p^{\prime}(w)=0$. Since the center $c$ of the disk $D(c, r)$ is the middle point of the segment joining $z_{1}$ and $z_{2}$, it is immediate to see that the maximum of the set

$$
\left\{\min \left\{\left|z-z_{1}\right|,\left|z-z_{2}\right|\right\}: z \in D(c, r)\right\}
$$

is achieved at two diametral points of $D(c, r)$, and by Pythagoras' Theorem this maximum is exactly

$$
\frac{\left|z_{1}-z_{2}\right|}{2} \sqrt{1+\cot ^{2}\left(\frac{\pi}{n}\right)}=\frac{\left|z_{1}-z_{2}\right|}{2} \frac{1}{\sin \left(\frac{\pi}{n}\right)} .
$$


Since we have $\left|z_{1}-z_{2}\right|=\omega(p)$, it follows that if $w$ is different from $z_{1}$ and from $z_{2}$, then

$$
\tau(p) \leq \min \left\{\left|w-z_{1}\right|,\left|w-z_{2}\right|\right\} \leq \frac{\omega(p)}{2 \sin \left(\frac{\pi}{n}\right)} .
$$

On the other hand, if $w=z_{1}$ or $w=z_{2}$, then

$$
\tau(p) \leq\left|z_{1}-z_{2}\right|=\omega(p) .
$$

It follows from (8) and (9) that the second inequality holds whenever $z_{1}$ and $z_{2}$ are simple roots of the polynomial $p$ or $2 \sin (\pi / n) \leq 1$, that is, whenever $z_{1}$ and $z_{2}$ are simple roots of the polynomial $p$ or $n \geq 6$. Since we assume that $p$ has two different roots, if $n=2$, the roots $z_{1}$ and $z_{2}$ are simple roots. Therefore, the second inequality in (6) holds for $n=2$. Note that if $n=2$ the inequality is trivially verified as $\omega(p) / 2=\tau(p)=\left|z_{1}-z_{2}\right| / 2$.

The above argument leaves open the cases of $n \in\{3,4,5\}$ in which $z_{1}$ or $z_{2}$ (chosen as above to satisfy $\left|z_{1}-z_{2}\right|=\omega(p)$ ) is not a simple root of $p$. Next we give a proof for each of these cases.

Let $n=3, \omega(p)=\left|z_{1}-z_{2}\right|$ and assume that $z_{1}$ is a double root and $z_{2}$ is a simple root of $p$. Clearly in this case $p$ has no other roots and therefore $p(z)=$ $\left(z-z_{1}\right)^{2}\left(z-z_{2}\right)$ (up to a constant multiple). A direct calculation shows that $p^{\prime}$ has roots $z_{1}$ and $\left(z_{1}+2 z_{2}\right) / 3$. Therefore $\tau(p)=\omega(p) / 3$, and the second inequality in (6) is true.

Let $n=4, \omega(p)=\left|z_{1}-z_{2}\right|$ and assume that $z_{1}$ has multiplicity $k_{1}$ and $z_{2}$ multiplicity $k_{2}$, with $k_{1}+k_{2} \geq 3$. By a result of Marden (see [3, Theorem (25,1)]), $p^{\prime}$ has at least one root $w$ (different from $z_{1}$ and $z_{2}$ ) in the disk $D(c, r)$, where

$$
c=\frac{z_{1}+z_{2}}{2}, \quad r=\frac{\left|z_{1}-z_{2}\right|}{2} \cot \left(\frac{\pi}{2 q}\right),
$$

where $q=n+1-k_{1}-k_{2}$. Note that the disc $D(c, r)$ may have a different radius from the one considered in the first part of the proof, but still the same argument applies to show that

$$
\tau(p) \leq \min \left\{\left|w-z_{1}\right|,\left|w-z_{2}\right|\right\} \leq \frac{\omega(p)}{2} \frac{1}{\sin \left(\frac{\pi}{2 q}\right)} .
$$

Since $n=4$ we have

$$
2 q=2\left(5-k_{1}-k_{2}\right) \leq 2(5-3)=4=n,
$$

meaning that

$$
\frac{1}{\sin \left(\frac{\pi}{2 q}\right)} \leq \frac{1}{\sin \left(\frac{\pi}{n}\right)} .
$$

The inequalities (10) and (11) yield the second inequality in (6).

The rest of the proof deals with the case $n=5$. Let $\omega(p)=\left|z_{1}-z_{2}\right|$ and assume that the multiplicity of $z_{1}$ as a root of $p$ is $k_{1}$ and the multiplicity of $z_{2}$ is $k_{2}$, with $k_{1}+k_{2} \geq 3$.

If $k_{1}+k_{2} \geq 4$, then, as before, the second inequality in (6) can be deduced from Marden's result [3, Theorem $(25,1)]$ since in this case we have $2 q=2\left(6-k_{1}-k_{2}\right) \leq$ $5=n$. 
Now consider the case when $k_{1}+k_{2}=3$ and assume that $z_{1}$ is a double root of $p$ and $z_{2}$ a simple root of $p$, and $p^{\prime}$ has no other root but $z_{1}$ in the disk $D(c, r)$, where

$$
c=\frac{z_{1}+z_{2}}{2}, \quad r=\frac{\left|z_{1}-z_{2}\right|}{2} \cot \left(\frac{\pi}{5}\right) .
$$

We need to prove that $\tau(p) \leq \omega(p) / 2 \sin (\pi / 5)$ (notice that $\tau(p) \leq \omega(p)$ is now trivial, but $2 \sin (\pi / 5)>1)$.

After rotation, scaling and translation, we only need to consider the case of

$$
p(z)=z^{2}(z-1)(z-\alpha)(z-\beta)
$$

where $\alpha, \beta$ are different from 0 and 1 , and $\omega(p)=1$, that is $|\alpha|,|\beta| \geq 1$. Put

$$
\mu:=\frac{1}{2 \sin (\pi / 5)}=\sqrt{\frac{2}{5-\sqrt{5}}} \approx 0.850650808352
$$

and note that the inequality we want to prove is just $\tau(p) \leq \mu$. We prove this by contradiction and henceforth assume that

$$
\tau(p)>\mu .
$$

Define the following "circular triangles" in the upper half-plane:

$$
\begin{aligned}
A & :=\operatorname{cl}(D(1 / 2,7 / 6) \backslash(D(0,1) \cup D(1,1))), \\
U & :=D(1 / 2, \sqrt{3} / 2) \backslash(D(0, \mu) \cup D(1, \mu)),
\end{aligned}
$$

where cl denotes the closure in $\mathbb{C}$. Let $A^{*}:=\{\bar{a}: a \in A\}$ and $U^{*}$ be the corresponding conjugate sets in the lower half-plane; see Figure 1.

Claim 1. The points $\alpha$ and $\beta$ cannot both be outside $A \cup A^{*}$.

Proof. Assume that both $\alpha$ and $\beta$ lie outside the region $A \cup A^{*}$. Apply Lemma 1 to the disk $D(1 / 2,1 / 2)$ and parameters $n_{1}=3, r_{1}=1 / 2, n_{2}=2$. The disk $D(1 / 2,1 / 2)$ contains exactly three roots of $p$ (the double root at 0 and the simple one at 1 ). Let $r_{2}$ be such that

$$
\min \{|\alpha-1 / 2|,|\beta-1 / 2|\} \geq r_{2}>\frac{7}{6} .
$$

Note that under these assumptions Lemma 1 implies that the circle $D(1 / 2,1 / 2)$ contains exactly two roots of $p^{\prime}$. This means that it must contain a non-zero root of $p^{\prime}$ (call it $v$ ). Now,

$$
\begin{aligned}
\tau(p) & \leq \min \{|v|,|v-1|\} \leq \sqrt{\left(\frac{1}{2}\right)^{2}+\left(\frac{1}{2}\right)^{2}} \\
& =\frac{\sqrt{2}}{2}<\mu,
\end{aligned}
$$

which contradicts our assumption $\tau(p)>\mu$.

Since our arguments in the rest of the proof are symmetric with respect to the line $\operatorname{Re}(z)=0$, in the following we will assume that

$$
\alpha \in A \text {. }
$$

Claim 2. The set $Z\left(p^{\prime}\right) \cap\left(U \cup U^{*}\right)$ is nonempty. 


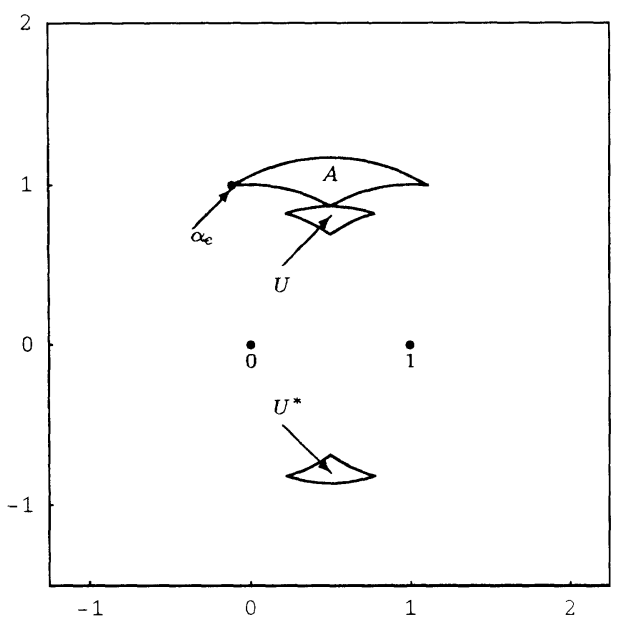

Figure 1. $A, U$ and $U^{*}$

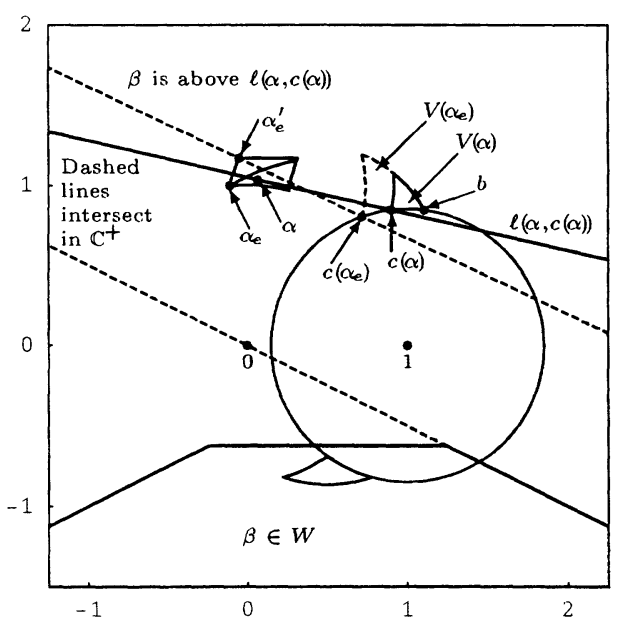

Figure 2. Claim 3

Proof. Applying again Marden's result [3, Theorem $(25,1)]$ to the double root of $p$ at $z=0$ and to the simple one at $z=1$, we obtain that there exists a nonzero root of $p^{\prime}$ inside the disk $D(1 / 2,(1 / 2) \cot (\pi / 6))=D(1 / 2, \sqrt{3} / 2)$. Since no such root can be at a distance $\leq \mu$ from 0 and 1 , the claim follows.

Claim 3. Let $W$ be the region that is bounded by and lies below the $\operatorname{lines} \operatorname{Im}(z)=$ $-5 \sqrt{5} / 18, \operatorname{Re}(z)+2 \operatorname{Im}(z)=0$ and $\operatorname{Re}(z)-2 \operatorname{Im}(z)=1$. Then $W$ does not contain any roots of $p^{\prime}$.

Proof. Assume that there exists a root $u$ of $p^{\prime}$ in $W$. Since the argument in the proof of this claim is symmetric with respect to the line $\operatorname{Re}(z)=1 / 2$, without loss of generality, we will let $\operatorname{Re}(\alpha) \leq 1 / 2$. Put

$$
A_{1}=\{z \in A: \operatorname{Re}(z) \leq 1 / 2\} \text {. }
$$


Since $u$ is in the convex hull of $Z(p)$ by Lucas' Theorem, we must have that $\beta$ lies in $W$. In particular, with $a:=(4+i 2 \sqrt{5}) / 9$, we have that

$$
|a-\beta|>\sqrt{5} / 2 \text {, }
$$

and therefore $\beta$ is outside the disk $D(a, \sqrt{5} / 2)$. Consider the disk $D(a, \sqrt{5} / 3)$, and observe that its boundary passes through the point 1 and through the leftmost point of region $A_{1}$, that is, the point $(-1+i 4 \sqrt{5}) / 9$. It is easy to see that region $A_{1}$ and point 0 are contained in $D(a, \sqrt{5} / 3)$. By Claim 1 , this means that $\alpha \in D(a, \sqrt{5} / 3)$. Applying Lemma 1 to the disk $D(a, \sqrt{5} / 3)$ with the following parameters: $n_{1}=$ $4, r_{1}=\sqrt{5} / 3, n_{2}=1, r_{2}=\sqrt{5} / 2+\epsilon$, where $\epsilon>0$ is chosen so that $|a-\beta|>\sqrt{5} / 2+\epsilon$, we conclude that $D(a, \sqrt{5} / 3)$ contains exactly three roots of $p^{\prime}$. Now, one of the three roots must be $z=0$, since it is a double root of $p$. As $\tau(p)>\mu$, the other two roots (say, $v_{1}, v_{2}$ ) must actually lie in the region

$$
V(\alpha):=D(a, \sqrt{5} / 3) \backslash(D(0, \mu) \cup D(1, \mu) \cup D(\alpha, \mu)) .
$$

The regions $V(\alpha), \alpha \in A_{1}$, have the same rightmost point. That is the point $b$ at the intersection of circles $D(a, \sqrt{5} / 3)$ and $D(1, \mu)$ :

$$
b:=\frac{1}{60}(45-3 \sqrt{5}+2 \sqrt{146+22 \sqrt{5}})+i \frac{1}{60}(6+6 \sqrt{5}+\sqrt{730+110 \sqrt{5}}) .
$$

This point is calculated using Mathematica's Solve command. All the calculations and the figures of this paper are done using Mathematica.

Consider the region $A_{1} \cap D(b, \mu)$. Since the maximum of the set

$$
\left\{|z-\zeta|: z \in A_{1} \cap D(b, \mu), \zeta \in V(\alpha)\right\}
$$

is attained at the point $b \in V(\alpha)$, and since we assume that $\tau(p)>\mu$, no root of $p$ lies in $A_{1} \cap D(b, \mu)$. Thus, we must have

$$
\alpha \in A_{1} \backslash D(b, \mu)=: A_{2} .
$$

Clearly $V(\alpha)$ lies entirely above the line through 1 and $\alpha$ for each $\alpha \in A_{2}$.

Let $t_{b}$ be the tangent line to the disk $D(1, \mu)$ at the point $b$. Define $\alpha_{e}:=$ $(-1+i 4 \sqrt{5}) / 9$ (this is the leftmost point of region $A$, and therefore of $A_{2}$ ). Comparing the slopes of the lines $\ell\left(\alpha_{e}, b\right)$ and $t_{b}$, we see that these two lines coincide. Thus, $t_{b}$ passes through the point $\alpha_{e}$. A calculation shows that the intersection of the circles $D(0,1)$ and $D(b, \mu)$ lies above $t_{b}$. Therefore, $A_{2}$ lies above $t_{b}$.

For $\alpha \in A_{2}$, define the point $c(\alpha)$ as the intersection of circles $D(\alpha, \mu)$ and $D(1, \mu)$ that has a larger imaginary part. Since $c(\alpha)$ is on the circle $D(1, \mu)$ it lies below $t_{b}$. Therefore, for each $\alpha \in A_{2}$ the line $\ell(\alpha, c(\alpha))$ separates the region $V(\alpha)$ from the triangle with vertices 0,1 and $\alpha$. By Lucas' Theorem, for a given $\alpha \in A_{2}$, $\beta$ has to lie above the line $\ell(\alpha, c(\alpha))$.

Next we prove that the slope $m(\alpha)$ of the line $\ell(\alpha, c(\alpha))$ is $>-1 / 2$ for each $\alpha \in A_{2}$. Instead of the region $A_{2}$ we will consider the larger region $A_{3}$ which is bounded by the unit circle from below, the line $\operatorname{Im}(z)=7 / 6$ from above, the arc of the circle $D\left(c\left(\alpha_{e}\right), \mu\right)$ from the left and by the arc of the circle $D(b, \mu)$ from the right; see Figure 2. For an arbitrary $\alpha \in A_{3}$ let $\alpha^{\prime} \in A_{3}$ be such that $\operatorname{Im}\left(\alpha^{\prime}\right)=7 / 6$ and the distance from $\alpha^{\prime}$ to $c(\alpha)$ is $\mu$. Clearly the slope of $\ell(\alpha, c(\alpha))$ is larger than the slope of the line $\ell\left(\alpha^{\prime}, c(\alpha)\right)$. Thus to get the minimum for the slopes of $m(\alpha)$ for $\alpha \in A_{3}$, it is sufficient to consider $\alpha$ 's on the the top edge of $A_{3}$ : this is a line segment and we call it $A_{4}$. Since the line segment joining $\alpha$ and $c(\alpha)$ has 
the constant length $\mu$, the slope $m(\alpha)$ is minimal (for $\alpha \in A_{4}$ ) when $c(\alpha)$ has the smallest imaginary part. Since $\operatorname{Im}\left(c\left(\alpha_{e}\right)\right) \leq \operatorname{Im}(c(\alpha))$ for all $\alpha \in A_{4}$, we conclude that the line $\ell\left(\alpha_{e}^{\prime}, c\left(\alpha_{e}\right)\right)$ has the minimum slope, which we calculate to be

$$
\frac{-21+4 \sqrt{5}+\sqrt{-10+18 \sqrt{5}}}{\sqrt{-349+\frac{912}{\sqrt{5}}+42 \sqrt{-10+18 \sqrt{5}}-8 \sqrt{-50+90 \sqrt{5}}}} \approx-0.473778>-\frac{1}{2} .
$$

Therefore, each line $\ell(\alpha, c(\alpha)), \alpha \in A_{2}$, intersects the line $\operatorname{Re}(z)+2 \operatorname{Im}(z)=0$ in $\mathbb{C}^{+}$; see Figure 2. Since we concluded that $\beta$ must be above the line $\ell(\alpha, c(\alpha))$ and since the half-plane above $\ell(\alpha, c(\alpha))$ does not intersect $W$, and we also concluded that $\beta \in W$, we have the desired contradiction.

Claim 4. The set $Z\left(p^{\prime}\right) \cap U$ is nonempty.

Proof. This claim is an immediate consequence of Claims 2 and 3, as all we need to verify is that $U^{*} \subset W$, where $W$ is the region defined in Claim 3. Now, $W$ and $U^{*}$ are both symmetric with respect to the line $\operatorname{Re}(z)=1 / 2$, and the topmost segment delimiting $W$ is wider than the interval $[0,1]$. Since, on the other hand, $U^{*}$ is narrower, all we need to check is that the topmost point $1 / 2-i \sqrt{\mu^{2}-1 / 4}$ of $U^{*}$ lies below the $\operatorname{line} \operatorname{Im}(z)=-5 \sqrt{5} / 18$. But this is immediate since $\sqrt{\mu^{2}-1 / 4}>$ $5 \sqrt{5} / 18$.

Claim 5. Let $\alpha \in A$. Then $\operatorname{Re}(\alpha)<0$ or $\operatorname{Re}(\alpha)>1$. If $\operatorname{Re}(\alpha)<0$, then there is a root $u$ of $p^{\prime}$ in $U$ such that $\operatorname{Re}(u)>1 / 2$, and if $\operatorname{Re}(\alpha)>1$, then there is a root $u$ of $p^{\prime}$ in $U$ such that $\operatorname{Re}(u)<1 / 2$.

Proof. By Claim 4 we know that there exists a root of $p^{\prime}$ in $U$. Consider the set

$$
B:=\{z \in A: 0 \leq \operatorname{Re}(z) \leq 1\} \text {. }
$$

Clearly the maximum of the set

$$
\{|z-\zeta|: z \in B, \zeta \in U\}
$$

is attained at the opposite corners of the sets $B$ and $U$ and can be calculated to be

$$
\sqrt{\frac{47}{18}-\frac{1}{2 \sqrt{5}}-\frac{1}{3} \sqrt{18+4 \sqrt{5}}} \approx 0.810705 \text {. }
$$

This number is smaller than $\mu$. Since we assume that $\tau(p)>\mu$, no roots of $p$ can be in $B$, thus $\operatorname{Re}(\alpha)<0$ or $\operatorname{Re}(\alpha)>1$. If $\operatorname{Re}(\alpha)<0$ and if $v \in U$ is such that $\operatorname{Re}(v) \leq 1 / 2$, then the distance between $\alpha$ and $v$ is less than $\mu$. Therefore, no such $v$ can be a root of $p^{\prime}$. Since there is a root of $p^{\prime}$ in $U$ it must have real part $\geq 1 / 2$. The case $\operatorname{Re}(\alpha)>1$ follows by a symmetric argument with respect to the line $\operatorname{Re}(z)=1 / 2$.

Put

$$
A_{l}=\{z \in A: \operatorname{Re}(z)<0\} \quad \text { and } \quad U_{r}:=\{u \in U: \operatorname{Re}(u)>1 / 2\} .
$$

Since our arguments in the rest of the proof are perfectly symmetric with respect to the line $\operatorname{Re}(z)=1 / 2$, we will henceforth assume that $\alpha \in A_{l}$. As a consequence of Claim 5 this implies that there is a root $u$ of $p^{\prime}$ in $U_{r}$. Note that the three corners of the region $A_{l}$ are

$$
-\frac{1}{9}+i \frac{4 \sqrt{5}}{9}, \quad i, \quad i \frac{\sqrt{10}}{3}
$$


and the corners of the region $U_{r}$ are

$$
\frac{1}{2}+i \frac{1}{2} \sqrt{\frac{1}{5}(5+2 \sqrt{5})}, \quad 1-\frac{1}{2 \sqrt{5}}+i \frac{1}{2} \sqrt{\frac{1}{5}(9+2 \sqrt{5})}, \quad \frac{1}{2}+i \frac{\sqrt{3}}{2} .
$$

In the following claim we will locate $\beta$, the fifth root of $p$. To do this, define the pair of concentric disks

$$
C_{1}=D(0, \sqrt{10} / 3), \quad C_{2}=D(0, \sqrt{10} / 2),
$$

let $\ell_{1}$ be the line passing through the points

$$
\frac{1}{2}+i \frac{1}{2} \sqrt{\frac{1}{5}(5+2 \sqrt{5})} \text { and } 1
$$

and let $\ell_{2}$ be the line parallel to $\ell_{1}$ through the point 0 , that is the line through the points

$$
0, \quad \text { and } \quad \frac{1}{2}-i \frac{1}{2} \sqrt{\frac{1}{5}(5+2 \sqrt{5})} \text {. }
$$

Since there is a root of $p^{\prime}$ in $U_{r}$ and the region $U_{r}$ lies above the line $\ell_{1}$, while the roots 0,1 and $\alpha$ of $p$ lie below or on $\ell_{1}$, Lucas' theorem implies that $\beta$ must be above the line $\ell_{1}$.

Claim 6. $\beta \in C_{2}$.

Proof. Let us assume the contrary, that is, $\beta \notin C_{2}$. Since $A_{l} \subset C_{1}$, Lemma 1 can be applied to the disk $C_{1}$, with $r_{1}=\sqrt{10} / 3, n_{1}=4, n_{2}=1$. Choosing any $r_{2}$ that satisfies

$$
|\beta| \geq r_{2}>\sqrt{10} / 2
$$

yields that $C_{1}$ contains exactly 4 of the roots of $p$ (since $|\alpha| \leq \sqrt{10} / 3$ ), while $\beta$ lies outside circle $D\left(0, r_{2}\right)$. Under these hypotheses the implication is that circle $C_{1}$ contains exactly 3 roots of $p^{\prime}$ (one of which is $z=0$ ). Since $\beta$ is above the line $\ell_{1}$, these roots must be above the line $\ell_{2}$. Thus they are in the half-disk of $C_{1}$ above the line $\ell_{2}$ and also outside of the disks $D(0, \mu)$ and $D(1, \mu)$. Call this region $T$. The region $T$ consists of two pieces: one in $\mathbb{C}^{+}$the other in $\mathbb{C}^{-}$; see Figure 3 . Note that the corners of the region $W$ are the points

$$
1-\frac{5 \sqrt{5}}{18}(2+i) \approx-0.24226-i 0.62113 \text { and } \frac{5 \sqrt{5}}{18}(2-i) \approx 1.24226-i 0.62113 \text {. }
$$

Since the intersection of $\ell_{2}$ with the circle $D(1, \mu)$ in $\mathbb{C}^{-}$is

$$
\frac{1}{2}-i \frac{1}{2} \sqrt{1+\frac{2}{\sqrt{5}}} \approx 0.5-i 0.688191
$$

and the intersection of the circles $C_{1}$ and $D(1, \mu)$ in $\mathbb{C}^{-}$is

$$
\frac{1}{180}(145-9 \sqrt{5})-i \frac{1}{18} \sqrt{\frac{1}{10}(1457+261 \sqrt{5})} \approx 0.693752-i 0.793611,
$$

we conclude that the entire piece $T \cap \mathbb{C}^{-}$is contained in the region $W$, which, by Claim 3 , is free from the roots of $p^{\prime}$. Thus, these roots must be in $T \cap \mathbb{C}^{+}$. The maximum of the set

$$
\left\{|z-\zeta|: z \in A_{l}, \zeta \in T \cap \mathbb{C}^{+}\right\}
$$




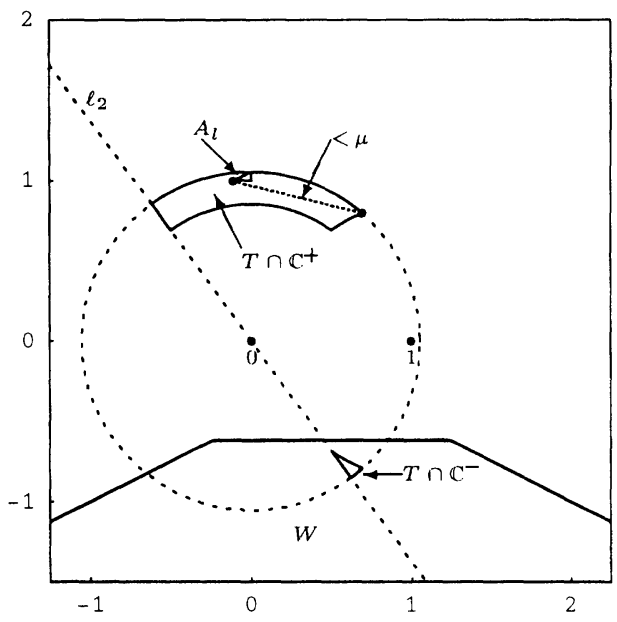

Figure 3. Claim 6

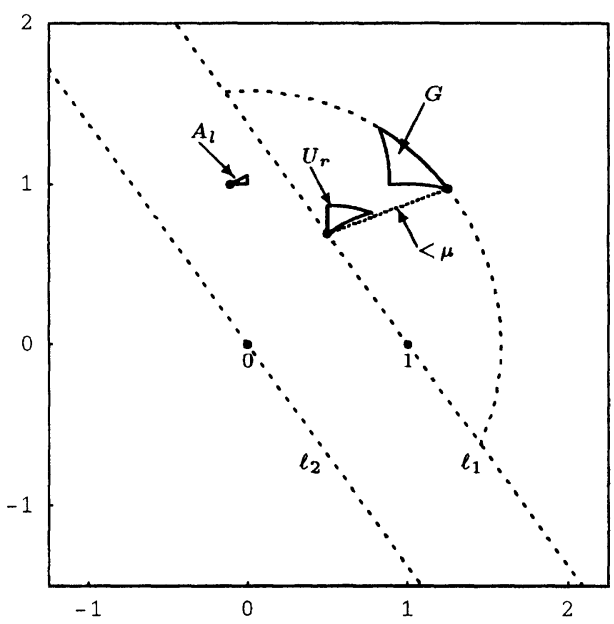

FIgURE 4. The final contradiction

is

$$
\frac{1}{9} \sqrt{\frac{371}{2}-\frac{9}{2 \sqrt{5}}-2 \sqrt{2914+522 \sqrt{5}}} \approx 0.829387<\mu .
$$

Thus, $T \cap \mathbb{C}^{+}$is free from the roots of $p^{\prime}$. Contradiction!

Next we arrive at the final contradiction of this proof. By Claim $6, \beta \in C_{2}$. Due to $\omega(p)=1, \beta$ is not allowed to be in the interior of $D(0,1) \cup D(1,1) \cup D(\alpha, 1)$. Therefore, since $\beta$ lies above $\ell_{1}$, it must be in the circular triangle which is inside $C_{2}$, outside $D(1, \mu)$ and $D(\alpha, \mu)$ and above the line $\ell_{1}$. Note that $\alpha \in A_{l}$ and that the disk $D(\alpha, \mu)$ changes with $\alpha$. It is easy to see that this circular triangle is largest when $\alpha$ is at the leftmost point of $A_{l}$, that is $\alpha=\alpha_{e}:=(-1+i 4 \sqrt{5}) / 9$. Let $G$ be the circular triangle inside $D(0, \sqrt{5 / 2})$, outside $D(1,1)$ and $D\left(\alpha_{e}, 1\right)$, and 
above the line $\ell_{1}$; see Figure 4 . The region $G$ is the largest allowable region for $\beta$. By Claim 5, there exists $u \in U_{r}$ which is a root of $p^{\prime}$. Considering the lowest corners of $G$ and $U_{r}$, we see that the maximum of the set

$$
\left\{|v-\gamma|: v \in U_{r}, \gamma \in G\right\}
$$

is

$$
\frac{1}{2} \sqrt{7+\frac{2}{\sqrt{5}}-\sqrt{15+6 \sqrt{5}}} \approx 0.800581 .
$$

This number is smaller than $\mu$. Thus $|u-\beta|<\mu$. Since $\beta \in Z(p)$ and $u \in Z\left(p^{\prime}\right)$, this implies that $\tau(p)<\mu$. Since we assumed that $\tau(p)>\mu$, we have reached the final contradiction and the theorem is proved.

\section{REFERENCES}

1. P. Borwein, T. Erdélyi, Polynomials and polynomial inequalities, Graduate Texts in Mathematics, 161, Springer-Verlag, 1995. MR 97e:41001

2. P. Henrici, Applied and computational complex analysis, Vol. 1, John Wiley \& Sons, 1988. MR 90d:30002

3. M. Marden, Geometry of polynomials, Second edition reprinted with corrections, American Mathematical Society, Providence, 1985. MR 37:1562

4. M. Mignotte, Mathematics for computer algebra, Springer-Verlag, 1992. MR 92i:68071

5. A. M. Ostrowski, On the moduli of zeros of derivatives of polynomials, J. Reine Angew. Math. 230 (1968), 40-50. MR 37:1563

6. J. L. Walsh, On the location of the roots of the derivative of a polynomial, C. R. Congr. Internat. des Mathématiciens, pp. 339-342, Strasbourg, 1920.

7. J. L. Walsh, The location of critical points of analytic and harmonic functions, Amer. Math. Soc. Colloq. Publ., Vol. 34, 1950. MR 12:249d

Department of Mathematics, Western Washington University, Bellingham, WashingTON 98225

E-mail address: curgus@cc.wwu.edu

Department of Mathematics, Western Washington University, Bellingham, WashingTON 98225

E-mail address: masciov@cc.wwu.edu 